\title{
Along-the-net reconstruction of hydropower potential with consideration of anthropic alterations
}

\author{
A. MASOERO, P. CLAPS, E. GALLO, D. GANORA \& F. LAIO \\ DIATI, Politecnico di Torino, Corso Duca degli Abruzzi 24, Torino, Italy \\ pierluigi.claps@polito.it
}

\begin{abstract}
Even in regions with mature hydropower development, requirements for stable renewable power sources suggest revision of plans of exploitation of water resources, while taking care of the environmental regulations. Mean Annual Flow (MAF) is a key parameter when trying to represent water availability for hydropower purposes. MAF is usually determined in ungauged basins by means of regional statistical analysis. For this study a regional estimation method consistent along-the-river network has been developed for MAF estimation; the method uses a multi-regressive approach based on geomorphoclimatic descriptors, and it is applied on 100 gauged basins located in NW Italy. The method has been designed to keep the estimates of mean annual flow congruent at the confluences, by considering only raster-summable explanatory variables. Also, the influence of human alterations in the regional analysis of MAF has been studied: impact due to the presence of existing hydropower plants has been taken into account, restoring the "natural" value of runoff through analytical corrections. To exemplify the representation of the assessment of residual hydropower potential, the model has been applied extensively to two specific mountain watersheds by mapping the estimated mean flow for the basins draining into each pixel of a the DEM-derived river network. Spatial algorithms were developed using the OpenSource Software GRASS GIS and PostgreSQL/PostGIS. Spatial representation of the hydropower potential was obtained using different mean flow $v$ s hydraulic-head relations for each pixel. Final potential indices have been represented and mapped through the Google Earth platform, providing a complete and interactive picture of the available potential, useful for planning and regulation purposes.
\end{abstract}

Key words hydropower potential, mean annual flow, anthropic changes

\section{INTRODUCTION}

Several research institutions are still investing time and effort in the search for diffuse renewable energy sources and in the discussion of their integration with the national systems (e.g. Lehrer et al. 2005). Even in regions with an existing and diffuse hydropower use, the compelling need for stable renewable power sources suggests the revision of plans of exploitation of water resources, while taking care of the environmental regulations. Up to this moment, studies have been generally conducted at a country level, considering therefore only the largest and most productive hydropower plants. The scale proposed here is concerned with the regional level and has a high (basin level) resolution, necessary for hydropower water resources planning in mountainous regions, particularly in the presence of existing derivations. Therefore, while assessing the residual potential, we take into consideration the hydrological effects of the current non-natural state of the river. Since studies on water resources are rarely developed in basins where anthropic impact is not negligible (see e.g. Savenije and Van der Zaag 2008), interactions between water systems and water availability are thus in need of further attention (Montanari et al. 2013) and motivate a considerable part of the activity documented here.

\section{METHODS AND APPLICATION FOR MAF ESTIMATION}

The analysis conducted here aims at increasing the detail of hydrological knowledge available in the Piemonte Region (northwest Italy), in the framework of the assessment of residual potential for hydropower generation. Gross hydropower potential is defined as $P=\gamma \cdot M A F \cdot H$, where $M A F$ is the mean annual flow, $H$ is the hydraulic head and $\gamma$ is the water specific weight.

In the first phase of the study, after a preliminary filtering to remove too short or unreliable time series, a dataset of 1566 station-years from 133 stations was established. In all of these stations, daily discharge data are available. The available hydrometric measures are related to partly altered hydrological systems, in which water intakes or water returns can produce a change in the main statistics of the streamflow. To evaluate the influence of alterations on natural streamflow caused by existing derivations, data on withdrawals for hydropower use were taken from a database of 350 hydropower plants located in the Piemonte Region. 
The first objective was to reconstruct the $M A F$ in the (hypothetical) situation of absence of derivations. To this aim, a new model of analytical correction is used, which allows reconstruction of the upstream "natural" mean discharge $\left(\bar{Q}_{U, \text { rec }}\right)$ from the available (altered) data. This has been done by defining a parameter $k$ as the rate between the maximum discharge that can be withdrawn $(\Delta Q)$ and the mean observed discharge $\left(\bar{Q}_{O}\right)$ :

$$
k=\frac{\Delta Q}{\bar{Q}_{O}}
$$

Using an exponential function to represent the average daily discharge distribution, an analytical correction method proposed by Masoero et al. (2014) shows that the upstream not-impacted (or "naturalised") mean discharge can be represented by the relationship:

$$
\bar{Q}_{U, \text { rec }}=\bar{Q}_{O} \cdot \frac{k}{\operatorname{ProductLog}[k]}
$$

where ProductLog $(x)$ is the inverse function of $x=\alpha e^{\alpha}$, with $\alpha$ as the exponential parameter. Therefore, a correct estimate of the upstream natural discharge can be provided knowing the parameter $k$ of each impacted section. Once the naturalised average $\left(\bar{Q}_{U, \text { rec }}\right)$ has been restored in all the available gauging sections, we proceeded to build the regional MAF estimation method. We consider the unaltered MAF in mm/year defined as MAF $=\bar{Q}_{U, \text { rec }} \cdot 31536 / A$, where $\bar{Q}_{U, \text { rec }}$ is the mean discharge value in $\mathrm{m}^{3} / \mathrm{s}, A$ is the basin area in $\mathrm{km}^{2}$ and 31536 is a conversion coefficient.

Many methodologies are available for the estimation of the mean annual flow in ungauged basins, and the differences that distinguish them are related to the amount of information available (e.g. Bocchiola et al. 2003, Blöschl et al. 2013). These methods typically include linear or nonlinear multiple regressions and hydrological simulations. The procedure adopted here is based on a linear multiple-regression approach (e.g. Kottegoda and Rosso 1997) where the dependent variable is the MAF (in mm) and the predictors are selected among 120 climatic, geological and morphometric descriptors available on the whole of the Piemonte Region (see the Atlas of Piemonte Rivers Basins, Gallo et al. 2013).

The procedure adopted is similar to the one proposed in the Spatially Smooth Estimation Method (SSEM) by Laio et al. (2011) presented for flood frequency regional analysis. The SSEM allows the mean discharge, as any other discharge-related statistics, to vary smoothly over the domain of analysis, without requiring homogeneous regions. In addition, our implementation of the SSEM procedure for the MAF estimation guarantees the congruence of the mean discharge along the stream, i.e. MAF downstream of a confluence is equal to the sum of the two MAF values recorded upstream. This result is obtained by using only raster-summable descriptors (i.e. weighted average values at the basin scale) as predictors in the regression model. As a consequence, only 24 descriptors out of 120 become eligible in the selection of the final regression model.

In order to build the regional model, we performed all the possible regressions combining from 1 to 4 (raster-summable) descriptors. Regressions coefficients are weighted to account for record length, then tested in their significance with the Student $t$-test, and for multicollinearity with the VIF test (e.g. Montgomery et al. 2001). The models presenting all-significant coefficients are sorted according to the related adjusted coefficient of determination $\left(\mathrm{R}^{2}{ }_{\text {adj }}\right)$. The best-performing models are finally checked again in detail to verify the regression model assumptions (e.g. residual normality, homoscedasticity). Performances are further evaluated with a leave-one-out crossvalidation. The final regression model we obtained is:

$\mathrm{MAF}=-7.3605 \cdot 10^{2}+1.2527 \cdot \mathrm{MAP}+3.2569 \cdot 10^{-1} \cdot h_{m}+5.2674 \cdot$ fourier $_{B 1}-6.7185 \cdot$ clc $_{2}$

where $M A P$ is the mean annual precipitation averaged at the basin scale, $h_{m}$ is the mean basin elevation, fourier $_{B I}$ is a phase parameter of the four coefficients used to represent the Fourier fitting of the basin-scale precipitation regime, and $c_{c_{2}}$ is the percentage of the basin area classified as 
"group 2" in the Corine Land Cover (European Environment Agency 2000). Descriptors in equation (3) are physically consistent with the physical drivers of the long-term water balance, such as precipitation, elevation and soil characteristics. The comparison between observed and estimated MAF values is reported in Fig. 1, with the different grey scales representing the different lengths of the available samples.

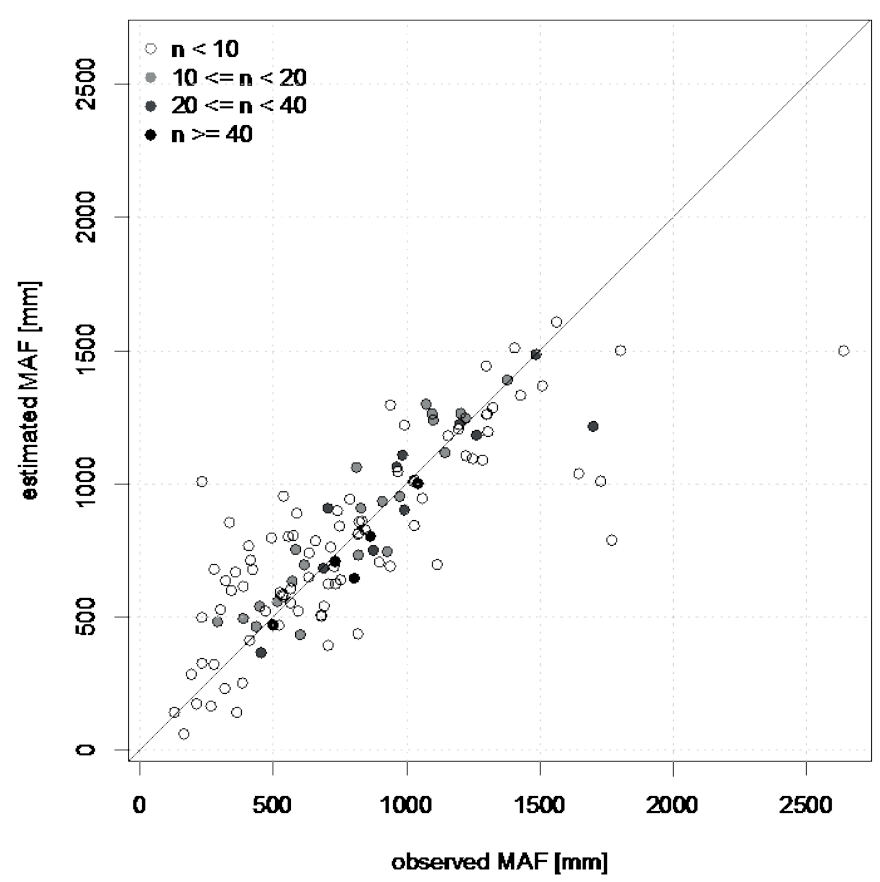

Fig. 1 Comparison between observed and estimated Mean Annual Flow (equation (4)); points shading is based on their sample size $n$.

\section{HIGH-RESOLUTION MAPPING OF HYDROPOWER POTENTIAL}

The regional MAF model described above was used to assess residual hydropower potential in two case-study basins, where the potential was required at high spatial resolution along streams. To this end the MAF was estimated along the whole river networks by applying extensively the regional model in the case-study basins to all network pixels considered as basin outlets (Fig. 2). Some basin details are reported in Table 1.

Table 1 Main characteristics of the case study basins.

\begin{tabular}{lllll}
\hline Basin & $\begin{array}{l}\text { Area } \\
\left(\mathrm{km}^{2}\right)\end{array}$ & $\begin{array}{l}\text { Network length } \\
(\mathrm{km})\end{array}$ & $\begin{array}{l}\text { Main-reach length } \\
(\mathrm{km})\end{array}$ & $\begin{array}{l}\text { Network pixels } \\
(\mathrm{n} .)\end{array}$ \\
\hline Chisone & 605 & 792 & 72 & 13204 \\
Stura di Demonte & 610 & 735 & 75 & 12198 \\
\hline
\end{tabular}

Flow directions for basin delineation have been reconstructed by GIS analysis, using a $50 \mathrm{~m}$ DEM and descriptors for the related thousands of sub-basins have been calculated through GRASSGIS analysis (e.g. Jasiewicz and Metz 2011). Given the large amount of basins to be considered, data were organized in a spatial database using PostgreSQL and PostGIS. Using this database, MAF values were estimated automatically in every pixel of the networks. From each of them the potential was assessed by guessing different hydraulic heads, as described later.

\section{Restoring of actual discharge}

The regional model of equation (3) provides estimates of the mean annual flow in natural, notimpacted conditions, as human impact was removed by means of equation (2). Nevertheless, water 


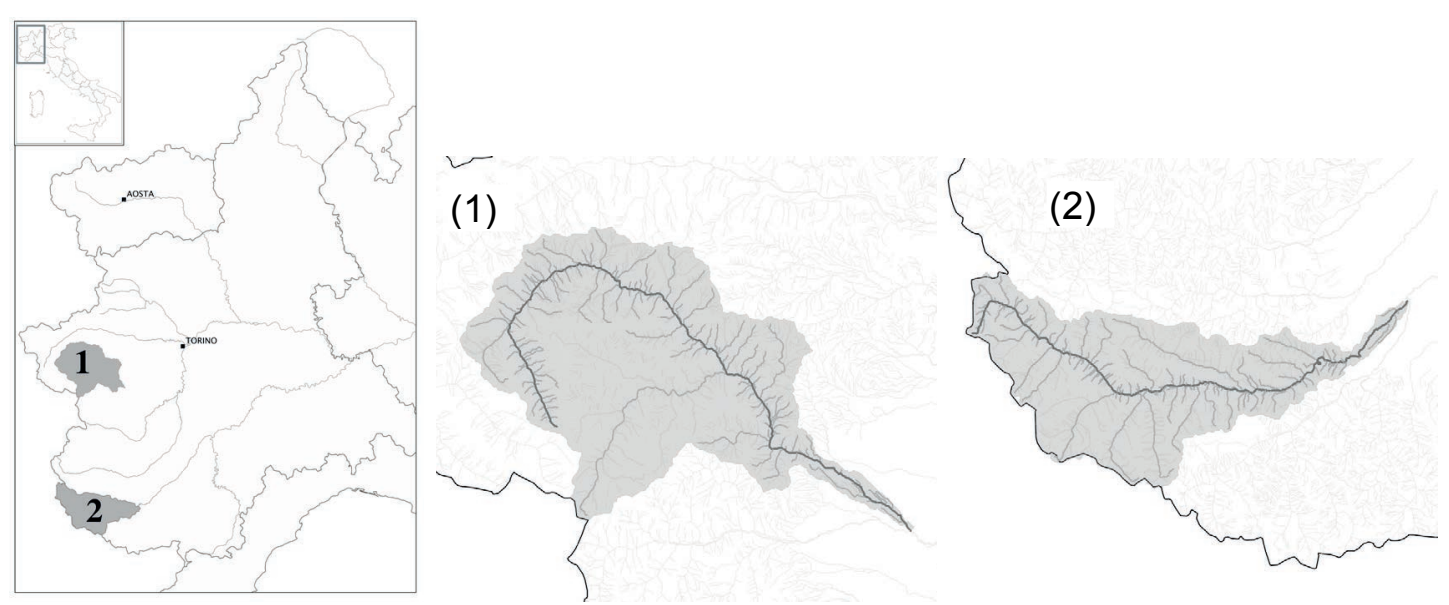

Fig. 2 Piemonte Region, in NW of Italy and case study basins. Chisone (1) and Stura di Demonte (2).

withdrawals for irrigation or hydropower generation must be taken into account in real-world basins. To assess the actual residual hydropower potential, we thus need to consider the impacts of these withdrawals on the estimated MAF, by adding the anthropic effect to the regional estimation. Therefore, all information about existing water intakes in the two case-study basins have been considered, adding irrigation intakes to the above-cited hydropower plant database.

Using the exponential form of the flow duration curve as above, the alteration can be accounted for by estimating the average withdrawn discharge $\bar{Q}_{W I T}$ as:

$$
\bar{Q}_{W I T}=\int_{0}^{\Delta Q} \exp \left(-\frac{Q}{\bar{Q}_{R E G}}\right)=\bar{Q}_{R E G} \cdot\left(1-\exp \left(\frac{\Delta Q}{\bar{Q}_{R E G}}\right)\right)
$$

where $\bar{Q}_{R E G}$ is the natural regional discharge and $\Delta Q$ is the maximum discharge that can be withdrawn. Considering also that derivation must preserve the Environmental Flow Discharge (EFD, e.g. Tharme 2003), the average value of the withdrawn discharge becomes:

$$
\bar{Q}_{W I T}=\int_{E F D}^{\Delta Q+E F D} \exp \left(-\frac{Q}{\bar{Q}_{R E G}}\right)=\bar{Q}_{R E G} \cdot \exp \left(-\frac{E F D}{\bar{Q}_{R E G}}\right)\left(1-\exp \left(\frac{\Delta Q}{\bar{Q}_{R E G}}\right)\right)
$$

The withdrawn discharge will be subtracted from the MAF estimates obtained at the water intakes sections, and from all sections downstream up to the point where water is returned to the river. In this way we represent the actual average flow along the net.

\section{Mapping the hydropower potential through an interactive GIS platform}

Hydrodynamic curve and hydropower potential network In a classical representation, the hydrodynamic curve of a basin is an Elevation-Contributing Area diagram that provides an initial assessment of the hydropower potential (Fig. 3(a)). The so-called "hydrodynamic value" is the area below the curve, where area counts as a MAF indicator. This representation is built along a single path, typically that of the main reach, and includes all tributaries. Once MAF is estimated over a grid, it is possible to build a more insightful hydrodynamic curve (i.e. Elevation - MAF) but also to devise a quasi-3D representation of the potential considering the entire network. This representation can be more significantly referred to as Hydropower Potential Network (HPN).

In order to have maps or synthetic representations of the HPN, for each network pixel point a definition of the hydraulic head should then be assumed, with immediate cautions related to the lack of consideration of hydraulic losses, of local feasibility conditions, etc. In this preliminary assessment we decided to limit the analysis to elevation drops coming from simple definitions.

A first instance of hydraulic head was obtained by searching return sections downstream of each node of the network using windows of fixed size centred in the derivation point. The radius of each 
window, where to search for the possible headraces, varied from $1 \mathrm{~km}$ to $5 \mathrm{~km}$. Distances were taken either using return point on the intersection of the circle with the network (Fig. 3(b)) or computing the distance $1-5 \mathrm{~km}$ along the network. HPN was then obtained by multiplying the derived elevation drop with the estimated MAF.
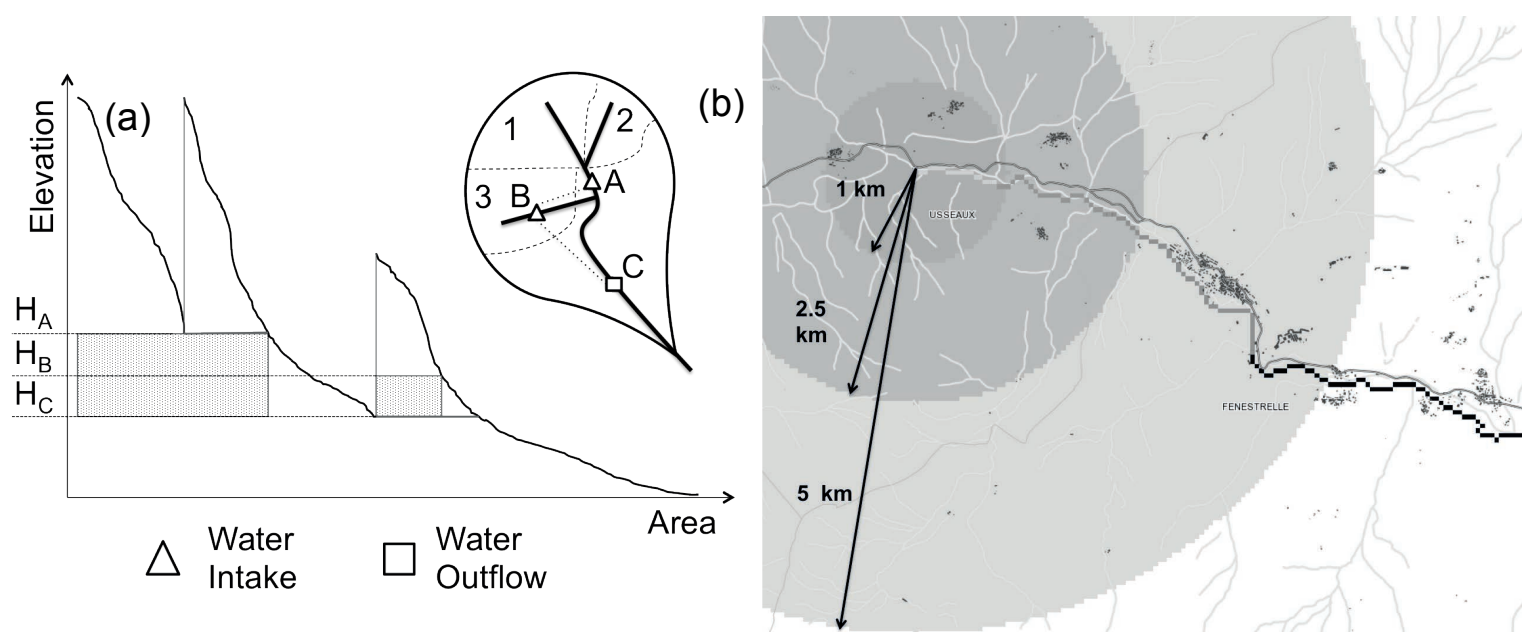

Fig. 3 (a) Classic Hydrodynamic Curve (HC). The grey area under the HC represents the hydrodynamic value that can be exploited by deriving in sections $\mathrm{A}$ and $\mathrm{B}$ and returning water in $\mathrm{C}$. The different peaks represent contributions from the main-channel tributaries. (b) Polar metric and curvilinear circular windows for possible headrace extraction.

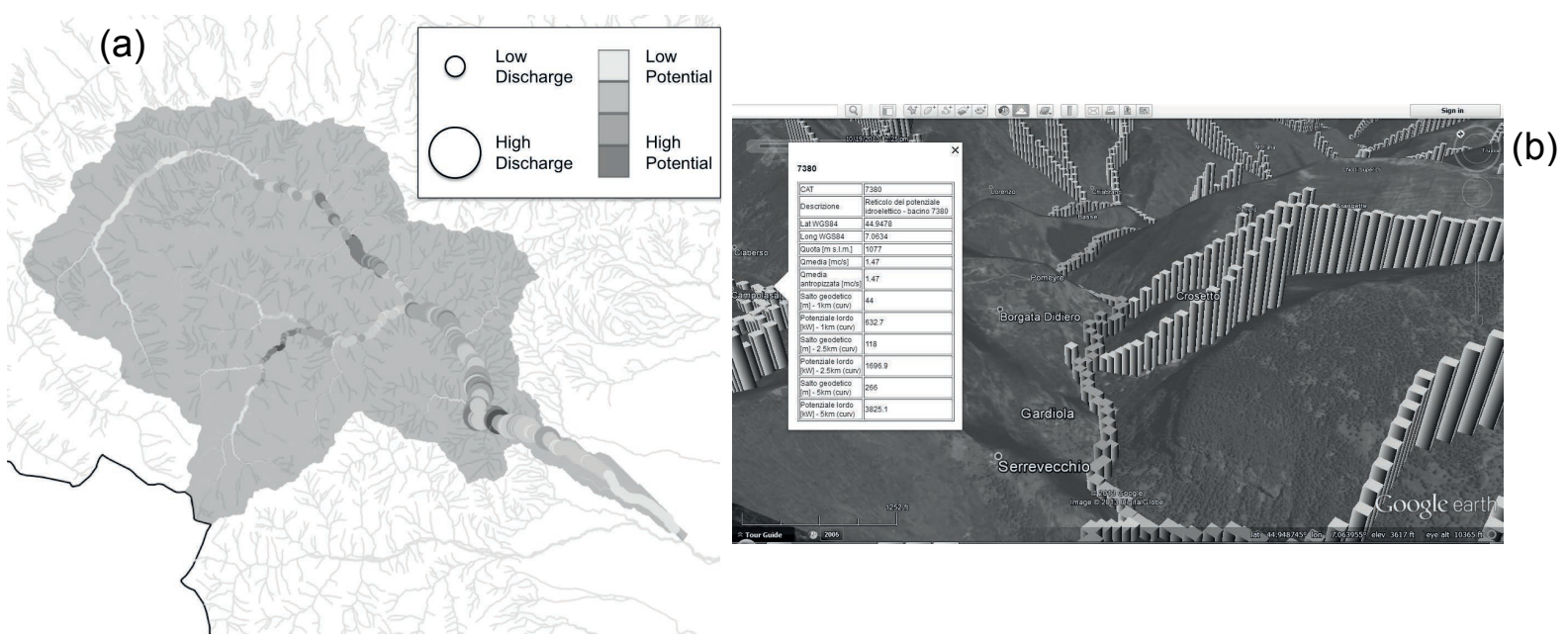

Fig. 4 Example of 2D (a) and 2.5D (b) representation of the Hydropower Potential Network.

\section{Representations of the Hydropower Potential Network: GIS and Google Earth}

The large amount and the complexity of the information built on the network requires specific attention to the representation techniques. Using a GIS desktop, which is typically two-dimensional, the representation of a third and a fourth variable (such as discharge, hydraulic head, potential) could be obtained by assigning different colours and sizes to the nodes of the grid (Fig. 4(a)). This kind of "static" map becomes insufficient when high resolution computation needs to be represented, rather addressing towards interactive maps The 2.5D representation used here (Fig. 4(b)) that allows consultation also to inexperienced GIS users, was devised using a $\mathrm{kmz}$ format for Google Earth, in which:

- data are represented in geographic coordinates;

- the considered nodes of the network are highlighted using prisms with a height equal to the hydraulic head; 
- the hydropower potential is represented by a colour scale assigned to the prisms, which varies from lighter tones (low potential) to darker ones (high potential);

- information on hydraulic head, MAF and potential can be shown as labels when the relevant information layers are individually activated; additional information can be viewed by querying the individual node.

\section{CONCLUSIONS}

Computation of the hydropower potential, as the product of MAF and a hypothetic hydraulic head, is addressed in a region with requirements of high-resolution representation in two mid-size basins. An improved multiple-regression MAF estimation method is first constructed, with constraints that produce congruent estimates along the network. Effects of water withdrawals are considered, with simplified analytical treatment, both in the data cleaning and in the estimation of actual MAF in basins downstream of derivation sections. With this prior knowledge we extensively mapped the residual hydropower potential (HP), associating MAF estimates with various hydraulic heads in every pixel of the river network of two case study basins, covering a $1000 \mathrm{~km}^{2}$ area. A new interactive tool was then built to represent the HP Network obtained, using Google-Earth and the underlying PostGIS database that manages the network pixels information.

Having tried to improve both hydrological methods and GIS tools for data analysis and representation, we offered a system for hydropower resources planning that is meant to be interactive, easy-to-use and that can be expanded in other regions with limited efforts.

\section{REFERENCES}

Blöschl, G., et al. (2013) Runoff Prediction in Ungauged Basins. Cambridge University Press.

Bocchiola, D., De Michele C. and Rosso, R. (2003) Review of recent advances in index flood estimation, Hydrology and Earth System Sciences 7(3), 283-296.

European Environmental Agency (2000) CORINE land cover technical guide - Addendum 2000, Technical Report n.40.

Gallo, E., et al. (2013) Atlante dei bacini imbriferi piemontesi (Atlas of Piemonte Region basins), RENERFOR Project Regione Piemonte, ISBN:978-88-96046-06-7.

Jasiewicz, J. and Metz, M. (2011) A new grass GIS toolkit for Hortonian analysis of drainage networks. Computers and Geosciences.

Kottegoda, N. T. and Rosso, R. (1997) Statistics, probability and reliability methods for civil and environmental engineers. McGraw-Hill.

Laio, F., et al. (2011) Spatially smooth regional estimation of the flood frequency curve (with uncertainty). Journal of Hydrology 408, 67-77.

Lehrer, B., Czisch, G. and Vassolo, S. (2005) The impact of global change on the hydropower potential of Europe: a model-based analysis. Energy Policy 33, 839-855.

Masoero, A., et al. (2014) A method to restore not-impacted flow duration curves statistics. Submitted paper

Montanari, A. et al. (2013). "Panta Rhei-Everything Flows": Change in hydrology and society - the IAHS Scientific Decade 2013-2022. Hydrological Sciences Journal 58, 1256-1275.

Montgomery, D., Peck, E. and Vining, G. (2001) Introduction to linear regression analysis. Wiley Series Probability and Statistics, third edn. Wiley, New York.

Savenije, H. and Van der Zaag, P. (2008) Integrated water resources management: Concepts and issues. Physics and Chemistry of the Earth, Parts A/B/C 33, 290-297.

Tharme, R. E. (2003) A global perspective on environmental flow assessment: emerging trends in the development and application of environmental flow methodologies for rivers. River Research and Applications 19(5-6), 397-441. 